\title{
PREMATURE RUPTURE OF MEMBRANES- A CLINICAL STUDY
}

\author{
S. Savitri', P. Padmaja², K. Lakshmi Prasanna ${ }^{3}$
}

${ }_{1}^{1}$ Assistant Professor, Department of Obstetrics and Gynaecology, Kurnool Medical College, Kurnool, Andhra Pradesh. ${ }^{2}$ Assistant Professor, Department of Obstetrics and Gynaecology, Kurnool Medical College, Kurnool, Andhra Pradesh. ${ }^{3}$ Assistant Professor, Department of Obstetrics and Gynaecology, Kurnool Medical College, Kurnool, Andhra Pradesh.

\begin{abstract}
BACKGROUND
ABSTRACT

Foetal and maternal risk proportionately increase with delay in time from occurrence of PROM to institution of treatment. We have undertaken a prospective study of term PROM cases mainly to evaluate maternal and perinatal outcome.

The objective of this study is to evaluate risk factors associated with term PROM and also its effects on maternal and perinatal outcome.
\end{abstract}

\section{MATERIALS AND METHODS}

100 pregnant women with term pregnancy of more than 37 weeks and before the onset of active phase of labour complaining of draining per vagina were included in the study. Delivery was planned in all cases, either by induction of labour or LSCS depending on the clinical findings at admission. Mode of delivery, maternal and perinatal morbidity were assessed.

\section{RESULTS}

Incidence of term PROM was 9.5\% of all deliveries. Cause of PROM was not known in majority of cases. Low socioeconomic status, cervicovaginal infections, anaemia, malpresentations and hydramnios were other contributing factors. Vaginal delivery was the commonest mode of delivery. Caesarean section was done in cases of failed induction and progress of labour, foetal distress, malpresentation and oligohydramnios. Maternal morbidity was due to intrapartum sepsis and perinatal morbidity and mortality resulting from neonatal sepsis and respiratory distress due to meconium aspiration.

\section{CONCLUSION}

Term premature rupture of membranes is one of the common and challenging problems in obstetric practice contributing to significant maternal and perinatal morbidity. Prophylactic antibiotics and timely induction of labour can reduce infectious morbidity in mother and the baby.

\section{KEYWORDS}

Premature Rupture of Membranes, Maternal Morbidity, Perinatal Morbidity, Oligohydramnios, Term.

HOW TO CITE THIS ARTICLE: Savitri S, Padmaja P, Prasanna KL. Premature rupture of membranes- a clinical study. J. Evolution Med. Dent. Sci. 2018;7(07):892-894, DOI: 10.14260/jemds/2018/203

\section{BACKGROUND}

Premature rupture of membranes is one of the most common complications of pregnancy that has major impact on foetal and maternal outcome. ${ }^{1}$ It is one of the common clinical problems, where an uneventful pregnancy may turn into a high-risk situation both for the mother and the baby. PROM is defined as undoubted spontaneous draining of liquor before recognisable onset of labour, whether at term or preterm. Term PROM complicates $5 \%-10 \%$ of pregnancies. ${ }^{1}$ Among these in $50 \%$ of cases there is spontaneous onset of labour within 12 hours, $70 \%$ within 24 hours, $85 \%$ within 48 hours and $95 \%$ within 72 hours. ${ }^{2}$ Foetal morbidities associated with term PROM include ascending infection and in-utero cord compression. ${ }^{3}$ Maternal morbidity is in the form of chorioamnionitis, placental abruption, endometritis and postpartum sepsis. ${ }^{1}$

'Financial or Other Competing Interest': None.

Submission 05-01-2018, Peer Review 30-01-2018,

Acceptance 06-02-2018, Published 12-02-2018.

Corresponding Author:

Dr. S. Savitri,

Assistant Professor

Suseela Netralaya, H. No. 46-89-10,

Budhawarpeta, Kurnool, Andhra Pradesh.

E-mail: drnehaperaka@gmail.com

DOI: $10.14260 /$ jemds/2018/203

(c) (i) $(5)$
The objective is to diagnose term PROM early and to effect successful vaginal deliveries with minimal foetal and maternal complications. Foetal and maternal risk proportionately increase with delay in time to presentation of the patients (from occurrence of PROM to institution of treatment). We have undertaken a prospective study of term PROM cases, mainly to evaluate maternal and perinatal outcome.

\section{Aims and Objectives of the Study}

1. To describe incidence and characteristics of patient with term PROM.

2. To evaluate risk factors.

3. To evaluate complications of PROM at term and its effect on maternal and perinatal outcome.

\section{MATERIALS AND METHODS}

It is a prospective interventional case study, where 100 pregnant women with term (> 37 weeks) pregnancy complaining of draining per vagina got admitted in Maternity Department of Govt. General Hospital, Kurnool, were selected for the study during August to November in 2006. Informed consent has been obtained from all the patients. The study is conducted according to Declaration of Helsinki and after Ethical Committee approval. 


\section{Criteria for Selection of Cases-}

1. Complaints of watery discharge per vagina after 37 weeks of pregnancy.

2. Demonstration of watery discharge on per speculum examination by keeping the patient in dorsal/ lithotomy position and asking the patient to cough.

3. Examination of the patient in standing position after giving a vaginal pad and looking for wetting of pad, eliminating other discharges.

4. Cervical dilatation of less than $3 \mathrm{~cm}$.

5. Ultrasound abdomen was done to assess Amniotic Fluid Index (AFI). 4

6. In doubtful cases, the following tests were conducted to confirm the presence of liquor amni in vagina. a) $\mathrm{PH}$ of fluid was assessed by litmus paper. ${ }^{5}$ Normal vaginal $\mathrm{PH}$ was seen as 4.5 to 5.5 , while that of liquor amni is 7 to 7.5 on the alkaline side. b) Crystalline/ Evaporation test ${ }^{6}$ - Fluid collected from vagina was allowed to dry on a clean glass slide. Presence of fern-like crystallisation as seen under high power microscope indicated evidence of liquor amni.

7. Culture and sensitivity of cervical swab was done in all cases prior to aseptic vaginal examination and in all cases prophylactic IV antibiotics were given. ${ }^{3}$

8. A fourth hourly record of maternal pulse, temperature, blood pressure and foetal heart rate was maintained. In a view to deliver the patient within 24 hours, induction of labour was planned in all cases except in those with malpresentations, severe oligohydramnios contracted pelvis and Cephalopelvic Disproportion (CPD). These cases were delivered by Lower segment Caesarean section (LSCS) soon after admission. Depending on the cervical score and favourability of cervix, labour was induced either with vaginal prostaglandin E2 gel7 or oxytocin drip. Labour was monitored with partogram and cases of foetal distress/ failed induction were delivered by caesarean section. Condition of the baby was noted immediately after delivery and resuscitation was done as necessary. Condition of both baby and mother was noted till the time of discharge.

\section{RESULTS}

During the study period from August 2006 to November 2006 , total number of deliveries was 1087. Out of this, 100 cases presented with PROM.

Incidence of PROM was $9.5 \%$ among all deliveries and more in unbooked cases- $870(80 \%)$ than in booked cases 217 (20\%). 20\% of cases had history of one or more abortions in previous pregnancies. 18\% had history of PROM in previous pregnancies. $9 \%$ had history of preterm delivery due to PROM. 12 cases had history of antecedent coitus. In majority of cases (51\%) cause of PROM was not known. Cervicovaginal infections (Table 1), malpresentations, hydramnios and cervical stitch were implicated risk factors (Table 2) in our study. Anaemia was present in $32 \%$ of cases. Among 48 primi gravidae, 33 delivered vaginally and 15 were delivered by LSCS. ${ }^{8}$ Among 52 multigravidae, 44 delivered vaginally and 8 cases by LSCS. ${ }^{8}$ Among 100 cases of term PROM, 8 cases were taken up for LSCS in view of CPD, malpresentation, severe oligohydramnios soon after admission. Remaining 92 cases, the Bishop score was assessed. Favourable cervical score was noted in 22 cases of primi and 35 cases of multi. Unfavourable cervical score was noted in 22 cases of primi and in 13 cases of multigravidae. Induction of labour was started by oxytocin drip in the former group and vaginal PGE2 gel was applied in the latter group. ${ }^{9}$ Induction delivery interval was less than 12 hours in $15 \%$ of cases, between 12 and 24 hours in $43 \%$ of cases and more than 24 hours in $32 \%$ of cases. In case of foetal distress, failed induction and progress of labour, baby was delivered by emergency LSCS.

\section{DISCUSSION}

PROM is one of the common and challenging problems in obstetric practice. Lack of consensus and clear evidence on optimal management of PROM at term has been a clinical issue and has gone through various cycles of masterly inactivity to immediate intervention.

Incidence of PROM at term was relatively high in women of low socio-economic group, who also happen to have poor nutritional status. ${ }^{10}$ No statistical significance was observed in its incidence in relation to parity. Age between 25 and 29 years showed maximum incidence of PROM at term. Incidence of PROM at term was $9.5 \%$ in this study, which is comparable with other studies. Unbooked cases showed higher incidence of PROM and compared to cases who had regular antenatal care.

Major risk factor for PROM was found to be maternal genital tract infection. $28 \%$ of cases had positive culture suggesting cervicovaginal infection. ${ }^{11}$ Coitus in the preceding few days is also implicated as a risk factor for PROM.12 Malpresentations, hydramnios and anaemia were other contributing factors.

In this study, total duration of labour in both primi and multigravidae was decreased. Duration of first stage of labour decreased, but duration of second stage remained unaltered. PROM results in release of prostaglandins, which in turn ripen the cervix and initiate uterine contractions. ${ }^{13}$

Vaginal delivery was the commonest mode of delivery in the present study (77 out of 100 cases). 23 cases were delivered by caesarean section. Failed induction and progress of labour was a common indication for LSCS (42\%) followed by foetal distress (29\%) and oligohydramnios (20\%). ${ }^{8}$ Failed induction and progress of labour could be due to relative uterine inertia resulting from intrapartum sepsis and unfavourable cervix (Table 3). Overall, there is four-fold increase in caesarean section rate in PROM. ${ }^{14}$ The rate of LSCS is $23 \%$ in present study. The rate of LSCS was two-fold high in women with unfavourable Bishop score at admission when compared to women with favourable Bishop score.

PROM increases the risk of intrapartum sepsis. Incidence of maternal morbidity was $15 \%$ (three-fold increase) in the form of puerperal pyrexia, wound infection and chorioamnionitis. 12 cases had puerperal pyrexia, which subsided in three to four days with appropriate antibiotics instituted after culture and sensitivity. ${ }^{15}$ In the present study, $6 \%$ perinatal mortality was noted because of neonatal septicaemia and respiratory distress due to meconium aspiration. ${ }^{16}$

Significant increase in neonatal morbidity with need for neonatal resuscitation requiring admission in neonatal intensive care unit was also observed in the present study. The hazard of infection increased proportionately with the time interval between rupture of membranes and delivery. 


\section{Abbreviations}

Premature Rupture of Membranes (PROM), Amniotic Fluid Index (AFI), Lower Section Caesarean Section (LSCS), Cephalopelvic Disproportion (CPD), PGE2 (Prostaglandin E2).

\section{CONCLUSION}

Premature rupture of membranes at term is a significant obstetric problem contributing to maternal morbidity and perinatal morbidity and mortality. Prophylactic antibiotics, timely induction of labour can reduce the infections in mother and also reduced neonatal intensive and special care admission.

\section{REFERENCES}

[1] Poma PA. Premature rupture of membranes. Journal of the National Medical Association 1996;88(1):27-32.

[2] Caughey AB, Robinson JN, Norwitz ER. Contemporary diagnosis and management of preterm premature rupture of membranes. Reviews in Obstetrics and Gynecology 2008;1(1):11-22.

[3] Seelbach-Goebel B. Antibiotic therapy for premature rupture of membranes and preterm labor and effect on fetal outcome. Geburtshilfe und Frauenheilkunde 2013;73(12):1218-27.

[4] Weissmann-Brenner A, O'Reilly-Green C, Ferber A, et al. Values of amniotic fluid index in cases of preterm premature rupture of membranes. J Perinat Med 2009;37(3):232-5.

[5] Ernest JM, Meis PJ, Moore ML, et al. Vaginal pH: a marker of preterm premature rupture of the membranes. Obstet Gynecol 1989;74(5):734-8.

[6] Schiøtz $H$. The evaporation test for detecting rupture of the fetal membranes. Acta Obstet Gynecol Scand 1987;66(3):245-6.
[7] Mahmood TA, Dick MJ, Smith NC, et al. Role of prostaglandin in the management of prelabour rupture of the membranes at term. $\mathrm{Br} \mathrm{J}$ Obstet Gynaecol 1992;99(2):112-7.

[8] Garite TJ. Management of premature rupture of membranes Clinics in Perinatology 2001;28(4):83747.

[9] Mozurkewich EL, Wolf FM. Premature rupture of membranes at term: a meta-analysis of three management schemes. Obstet Gynecol 1997;89(6):1035-43.

[10] Ferguson SE, Smith GN, Salenieks ME, et al. Preterm premature rupture of membranes. Nutritional and socioeconomic factors. Obstet Gynecol 2002;100(6):1250-6.

[11] Nakubulwa S, Kaye DK, Bwanga F, et al. Genital infections and risk of premature rupture of membranes in Mulago Hospital, Uganda: a case control study. BMC Research Notes 2015;8:573.

[12] Flood B, Naeye RL. Factors that predispose to premature rupture of the fetal membranes. JOGN Nurs 1984;13(2):119-22.

[13] O'Brien WF. The role of prostaglandins in labor and delivery. Clin Perinatol 1995;22(4):973-84.

[14] Kalem NM, Köşüş A, Kamalak Z, et al. Factors affecting the rates of caesarean sections in cases with premature rupture of membranes (PROM) at term. J Obstet Gynaecol 2017;37(5):585-90.

[15] Flenady V, King J. Antibiotics for prelabour rupture of membranes at or near term. Cochrane Database Syst Rev 2002;(3):CD001807.

[16] Nili F, Ansari AAS. Neonatal complications of premature rupture of membrane. Acta Med Iran 2003;41(3):175-9. 